\title{
Future of Nano science in Technology for Prosperity: A Policy Paper
}

\author{
Harikamal Barman ${ }^{1 *}$, Shaon Kr Das ${ }^{2}$ and Aniruddha Roy ${ }^{3}$ \\ ${ }^{1}$ University of North Bengal, Darjeeling-734013, West Bengal, India \\ ${ }^{2}$ ICAR RC for NEH Region, Sikkim Centre-737102, Sikkim, India \\ ${ }^{3}$ ICAR RC for NEH Region, Umiam-793103, Meghalaya, India
}

\begin{abstract}
Received: December 13, 2017; Accepted: December 23, 2017; Published: January 12, 2018
*Corresponding author: Harikamal Barman, University of North Bengal, Darjeeling-734013, West Bengal, India, E-mail: harikamalbarman@ gmail.com
\end{abstract}

\begin{abstract}
TIn the process of miniaturization nanotechnology has unleashed enormous prospects for the development of new products and applications for a wide range of industrial and consumer sectors. Currently the most commonly investigated nanomaterials are variants of nanorobots, nanocrystals, Dendrimers, Nanopore sensors, quantum dots, and carbon based materials (e.g., fullerenes, nanotubes). While the source elements are often the same as the ions already used in commercial products, nanomaterials are highly reactive and often differ in many physical and chemical characteristics than their ionic counterparts. These different characteristics make them suitable for improvement or replacement of commercial products and applications. The current and projected applications of engineered nanomaterials span a wide range of sectors. These include cosmetics and personal care products; pesticides and fungicides, lubricants and fuel additives; paints and coatings; agrochemicals, plant protection products, and veterinary medicines, plastics, and weapons and explosives. More than 140 companies worldwide have already engaged in manufacture of nanomaterials. The concerns due to emergence of nanotechnology include health and safety, environmental, analytical, ethical, policy and regulatory issues. While it is often difficult to predict the future, some things seem inevitable. Just as a ball thrown into the air can be expected to fall to the ground, so we can expect our technology to reach the molecular scale.
\end{abstract}

Keywords: Nanotechnology; Nano robots; Nano crystals; Nanopore sensors; Quantum dots

\section{Introduction}

The term "Nanotechnology" was defined by Tokyo Science University Professor Norio Taniguchi in a 1974 as follows: Nanotechnology' mainly consists of the processing of, separation, consolidation, and deformation of materials by one atom or by one molecule [1]. Engineered nanomaterials, developed using nanotechnology tools, can be defined as materials with dimensions between 1 and 100 nanometers in at least one dimension and possessing unique properties (magnetic, photonic, electronic, etc.). Imagine a supercomputer a billion times more powerful than today's and yet so small it would be barely visible by a light microscope [2]. Clean factories manufacturing without having to worry about pollution choking up the environment.
Cheap and abundant solar energy replacing conventional fossil fuels like oil, coal and gas. Building materials that are stronger, lighter and cheaper than the ones used in today's rockets, making lunar vacations no more expansive than says a trip to South Pole. A world where material abundance for all the people becomes a reality. Sounds too good to be true? Not for the new breed of scientists who believe that the 21st centuary could see all these science fiction dreams come true thanks to nanotechnology, a hybrid of chemistry and engineering that has opened up a whole new world of possibilities which If taken to their logical conclusion would completely change us and the world as we know it today [3]. Indeed, so exciting are the prospects of this revolutionary science that countries all over the world are investing in the research and development of nanotechnology. Clearly nanotechnology is slowly but surely capturing the attention of the scientific community, the media and no the public [4]. But just what exactly is nanotechnology and why everyone talking about it?

\section{Tools and Techniques}

\section{Scanning Electron Microscopy (SEM)}

SEM is an electron microscope that images the sample surface by scanning it with a high energy beam of electrons. Conventional light microscopes use a series of glass lenses to bend light waves \& create a magnified image while the SEM creates the magnified images by using electrons instead of light waves.

\section{Environmental Scanning Electron Microscopy (ESEM)}

While using the environmental scanning electron microscope (ESEM), it is not necessary to make nonconductive samples conductive. Materials samples do not need to be desiccated and coated with gold-palladium, for example, and thus their original characteristics may be preserved for further testing or manipulation.

\section{Transmission Electron Microscopy (TEM)}

TEM is a microscopy technique whereby a beam of electrons is transmitted through an ultra-thin specimen \& interacts as 
passes through the sample. An image is formed from the electrons transmitted through the specimen, magnified \& focused by an objective lens \& appears on an imaging screen. The contrast in a TEM image is not like the contrast in a light microscope image. In TEM, the crystalline sample interacts with the electron beam mostly by diffraction rather than by absorption.

\section{Atomic Force Microscopy (AFM)}

AFM is ideal for both qualitatively \& quantitatively measuring the nanometer scale surface roughness \& for visualizing the surface nano-texture on many types of material surfaces including polymer nano-composites \& nano-coated materials. Advantages of the AFM for such applications are derived from the fact that the AFM is non-destructive technique $\&$ it has a very high $3 \mathrm{~d}$ spatial resolution

\section{Scanning Tunneling Microscopy (STM)}

STM is an instrument for producing surface images with atomic scale lateral resolution, in which a fine probe tip is scanned over the surface of a conducting specimen, with the help of a piezoelectric crystal at a distance of $0.5-1 \mathrm{~nm}$, \& the resulting tunneling current or the position of the tip required to maintain a constant tunneling current is monitored.

\section{Assemblers}

A Nanoscale device which can be programmed to build more complex nanomachines. It is a tiny machine that is capable of self replication. It is inexpensive [5]. By ensuring that each atom is properly placed, they will manufacture products of high quality and reliability.

\section{Atomic Force Microscopy}

Measures the interaction force between the tip and surface. An atomically sharp tip is scanned over a surface with feedback mechanisms that enable the piezoelectric scanners to maintain the tip at a constant force (to obtain height information), or height (to obtain force information) above the sample surface [6]. Tips are made from $\mathrm{Si} 3 \mathrm{~N} 4$ or $\mathrm{Si}$, and extended down from the end of a cantilever.

\section{Scanning Tunneling Microscope}

Provides a three-dimensional profile of the surface to obtain atomic-scale images of metal surfaces, uses the quantum tunneling effect to view and manipulate Nanoscale particles, atoms and small molecules and to map surfaces [7]. It measures a weak electrical current flowing between tip and sample as they are held a very distance apart.

\section{Near Field Scanning Microscope}

Scans a very small light source very close to the sample. Detection of this light energy forms the image. NSOM can provide resolution below that of the conventional light microscope.

\section{Nanostructures}

The materials having at least one dimension are between 1
- $100 \mathrm{~nm}$. Different types of nanostructures are 2-D structures (1-D confinement): thin films and superlattices; 1-D structures (2-D confinement): nanowires, quantum wires, nanorods and nanotubes; 0-D structures (3-D confinement): nanoparticles and quantum dots; Special nanomaterials: bulk nano crystalline films and nanocomposites.

\section{Nano Factory}

Proposed system in which hypothetical nanomachines combine reactive molecules via mechanosynthesis to build larger atomically precise parts. These in turns are assembled by positioning mechanisms of assorted sizes to build macroscopic (visible) but still atomically precise products [8]. A nanofactory will be the end result of a convergence between nanotechnology (molecular scale engineering), rapid prototyping, and automated assembly.

\section{Nano Particle}

Spherical or capsule-shaped structure. Most are hollow, which provides a central reservoir that can be filled with anticancer drugs, detection agents, and chemicals, known as reporters, which can signal if a drug is having a therapeutic effect [9]. The surface of a nanoparticle can also be adorned with various targeting agents, such as antibodies, drugs, imaging agents and reporters. Ammonium citrate (aqueous) and imidazoline or oleyl alcohol (no aqueous) are additives for deagglomeration.

\section{Bucky Ball or Fullerene or $\mathrm{C}_{60}$}

Spherical shape with a hollow interior made of 20 hexagons and 12 pentagons. Fullerenes are molecular with exact no. of (60, $70,120,180$ ) of carbon atoms. Buckyballs, because they resemble the geodesic domes built by architect Buckminster Fuller. Discovered in 1985 among the byproducts of laser vaporization of graphite in which the carbon atoms are arranged in sheets [10]. Robert F. Curl Jr. and Richard E. Smalley, both of Rice University in Houston, Texas and Harold W. Kroto of the University of Sussex in England, won the 1996 Nobel Prize for Chemistry for their discovery of buckminsterfullerene, the scientific name for buckyballs [11]. Inhibiting the HIV by attaching fullerenes to the virus and thus preventing its replication.

\section{Carbon Nano Tubes}

Carbon atom form extended hollow tubes (cylindrical) instead of closed, hollow spheres. Carbon nanotubes can also form as a series of nested, concentric tubes. Carbon nanotubes can be used as nanometer-scale syringe needles for injecting molecules into cells and as Nanoscale probes for making fine-scale measurements [12]. Carbon nanotubes can be filled and capped, forming Nanoscale test tubes or potential drug delivery devices. Carbon nanotubes can also be "doped," or modified with small amounts of other elements, giving them electrical properties that include fully insulating, semiconducting, and fully conducting [13]. Chemically much less reactive than carbon atoms. Electrical conductivity of graphite, but conduct electricity along one axis rather than in all directions. Strength of diamond [14]. 


\section{Nano Robot}

Nano technological robot nano machine, which is a mechanical or electrochemical device whose dimensions are in $\mathrm{nm}$. To identify pathogens and toxins from samples of body fluid. Detection of toxic chemicals and the measurements of their concentrations in the environment.

\section{Nano Capsules}

Used as smart drugs or nano-encapsulation those have specific chemical receptors and only bind to specific cells. Higher dose loading with smaller dose volumes. Longer site-specific dose retention. More rapid absorption of active drug substances. Increased bioavailability of the drug. Higher safety and efficacy.

\section{Nano Crystals}

Nano crystals are aggregates of few hundred to tens of thousands of atoms that combine into a crystalline form of matter known as a "cluster". The first atomic-scale images of nanocrystals that help to reduce pollution show a surprising triangular, rather than hexagonal shape [15]. Used to make super-strong and longlasting metal parts. The crystals also might be added to plastics and other metals to make new types of composite structures.

\section{Nano Wires}

Solid "one dimensional". Can be conducting, semiconducting insulating can be crystalline, low defects. Nanotube defined - a long cylinder with inner and outer nm-sized diameters. Nanowire defined - a long, solid wire with nm diameter [16].

\section{Nanopore Sensor}

Rapid DNA sequencing, separation of single stranded and double stranded DNA in solution. Determination of length of polymers and separation of polymers by length. Potential applications for NASA missions including astronaut health, life detection and decoding of various genomes [17]. An engineered DNA strand between metal atom contacts could function as a molecular electronics device. Understanding the complex quantum physics via simulation guides design.

\section{Nano Composites}

Nanocomposite - consists of two or more synthesized materials of which at least one has Nanoscale dimensions. Multiple material possibilities (Organic + organic, Organic + inorganic, Inorganic + inorganic) and Nanoparticles or nanowire or nanotube + matrix material [18].

\section{Nano Wire}

Nano wires are built atom by atom on a solid surface. They can be coated with molecules viz. antibodies that will bind to proteins and other substances of interest to researchers and clinicians [19]. Nano wires are incredibly sensitive to such binding events and respond by altering the electrical current flowing through them and thus form the basis of ultra sensitive molecular detectors [20]. To create electrical circuits and semiconductor nano wire crossings, future of digital computing.

\section{Application of Nanotechnology}

\section{Aerospace}

Structural materials (e.g. saving weight and energy by using light-weight, ultra rigid materials). Information and communications technology e.g. more efficient design of data transfer between space vehicles and terrestrial information networks using electronic and optoelectronic nanotechnology components [21]. Sensorics (e.g. improving medical monitoring of astronauts with sensors based on nanostructured materials) and Thermal protection and control (e.g. improving thermal control systems through nanostructured diamond-like carbon coatings).

\section{Security and Defence}

Nanoscale electronic, improved sensory capabilities, enhanced computing power, storage capacity and electromechanical components could make control and steering of vehicles more effective and robust. Unmanned and autonomous systems in air, sea and space could be further reinforced [21]. Development of Nanoscale powders for use in propellants and explosives, enhancing the energy yield and speed of explosion.

\section{Catalysis, Chemistry and Materials Synthesis}

Chemical industry: catalysis (gold nanoparticles), pigments, coatings and lubricants, micro / nanoreaction technology and pharmaceuticals and cosmetics. Supramolecular host-guest structures are opening up synthesis routes in organic chemistry. Regioselectivity and stereo selectivity of catalysts can be increased. The higher surface to volume ratio means that much more of the catalyst is actively participating in the reaction [21]. Surface active membranes, nonporous (bio) filters and adsorption agents can be optimized. E.g. for sewage treatment, pollutant removal and byproduct separation.

\section{Food Security}

Cleaner agriculture and more targeted, preventative treatment. Networks of Wireless Nano sensors: to monitor (e.g., the presence of plant viruses or the level of soil nutrients). Near real time pathogen detection and location reporting by integrating Nano-electromechanical systems (NEMS) with new chip designs, without the need to resort to the widespread use of pesticide. Smart Field System that automatically detects locates reports and applies water, fertilizers (nanoparticles) and pesticides - going beyond sensing to automatic application, harvest timing, water quality measurement and control [22].

\section{Biomedical Applications of Nanotechnology}

Premise relatively small size, ease of transport within tissues/organs, ability to cross plasma membranes and potential target of biologically active molecules, will facilitate biomedical applications of nanoparticles in health and disease. About half of all pharmaceutical production will be dependent on nanotechnology affecting over $\$ 180$ billion in revenues in 10 15 years [22]. Nanotechnology will expand life spans, improve quality of health and enhance human physical capabilities. 


\section{Dendrimer}

A Dendrimer is a tree-like highly branched polymer molecule (Greek dendra $=$ tree), also called artificial protein. Dendrimers are synthesized from monomers with new branches added in discrete steps ("generation") to form a tree-like architecture. A high level of synthetic control is achieved through step-wise reactions and purifications at each step to control the size, architecture, functionality and monodispersity [23]. Dendrimer used for cancer or tumor-targeting agents, imaging contrast agents to pinpoint tumors, drug molecules for delivery to a tumor and reporter molecules that might detect if an anticancer drug is working.

\section{Liposome}

A type of nano particle made of lipids, or fat molecules, surrounding a water core. Liposome, several of which are widely used to treat infectious diseases and cancer, were the first type of nano particle to be used to create therapeutic agents with novel characteristics

\section{Information Technology}

Information technology revolution brought by miniaturization of silicon transistors. Further miniaturization possible: Fundamental physical limit of transistor dimension $10-20 \mathrm{~nm}, 10$ to 15 years. Future breakthroughs will likely come from nanotechnology: Carbon nano tube transistors: smaller and faster. Single electron transistors: quantum computers. Molecular scale devices or nano scale transistors, based on chemical self-assembly, will lead future advances in information technology.

\section{Nano-Optics and Information Storage}

Photonic crystals for multiplexing and all optical switching in optical networks. Atomically thin layers of nanostructure material used to substantially increase the information storage density.

\section{Consumer Products}

Nanoscale powders, in their free form, without consolidation or blending, used by cosmetics manufacturers: Titanium Dioxide and Zinc Oxide powders for facial base creams and sunscreen lotions. Iron Oxide powders as base material for rouge and lipstick. Improved wear and corrosion resistance. Nanocomposite materials, with increased impact strength, for automobiles.

\section{Textile Industry}

Integration of nano robots to give an article of clothing the capability to mend itself. Improve characteristics and functions such as crease resistance, breathing properties, wear resistance, spot and water repellence, antistatic properties, active ingredient storage or fire protection.

\section{Nano Crystals}

Nano crystals are aggregates of anywhere from a few hundred to tens of thousands of atoms that combine into a crystalline form of matter known as a "cluster". The first atomic-scale images of nanocrystals that help reduce pollution show a surprising triangular, rather than hexagonal shape. Used to make superstrong and long-lasting metal parts. The crystals also might be added to plastics and other metals to make new types of composite structures for everything from cars to electronics [23].

\section{Quantum Dot (Qdots)}

Semiconductor crystal with a diameter of few nanometers. Being quasi-zero dimension, quantum dots have a sharper density of states than higher dimensional structures. They have superior transport and optical properties, and are being researched foe use in diode lasers and detectors. Solid-state quantum computation. By applying small voltage to the leads, one can control the flow of electrons through the quantum dot and thereby make precise measurements of the spin and other properties therein. Because of their small size, quantum dots can function as cell and even molecule specific markers.

\section{Future Lies in Nanotechnology}

Nanotechnology would give us an opportunity, if we take appropriate and timely action to become one of the important technological nations in the world. The world market in 2005 is for nano materials, nano tools, nano devices and nano biotechnology, which put together, is expected to be over hundred billion dollars. Nanotechnology is a new technology that is knocking at doors [23].

\section{Conclusions}

Due to its highly reactive surface area, Nanoparticles has very high surface adsorption properties which can be executed for different purposes. Due to high and irreversible adsorption of toxic metals on the nanoparticles, they can be used for remediation of metal contaminated soils and water. Nanomaterials can be excellently used for production of cementing or coating agents for controlled or slow release fertilizers, they are proven superior to other coating agents. Nanomaterials can be excellently used for production of cementing or coating agents for controlled or slow release fertilizers, they are proven superior to other coating agents. As the size decreases computer will compute faster, materials will be stronger, medicine will cure more diseases.

\section{References}

1. Pavel A Troshin, Rimma N Lyubovskaya, Ilya N Ioffe, Natalia B Shustova, Erhard Kemnitz, Sergey I Troyanov. Synthesis and Structure of the Highly Chlorinated [60] Fullerene C60Cl30 with a Drum-Shaped Carbon Cage. Chem. Int. Ed. Engl. 2004;44:234-237. doi: 10.1002/ anie. 200461531

2. Das Shaon Kumar. Chemicals Responsible for Systemic Acquired Resistance in Plants-A Critical Review. Journal of Atoms and Molecules. 2014;4(3):45-51.

3. Sergey I Troyanov, Natalia B Shustova, Alexey A Popov, Lev N Sidorov, Erhard Kemnitz . Preparation and Structural Characterization of Two Kinetically Stable Chlorofullerenes, $\mathrm{C} 60 \mathrm{Cl} 28$ and $\mathrm{C60Cl30.} \mathrm{Chem.} \mathrm{Int.}$ Ed. 2005;117(3):436-439.

4. Harikamal Barman, Aniruddha Roy, Shaon Kumar Das. Evaluation of Plant Products and Antagonistic Microbes Against Grey Blight (Pestalotiopsis Theae), A Devastating Pathogen Of Tea. African Journal of Microbiology Research. 2015;9(18):1263-1267. 
5. Meital Reches, Ehud Gazit. Casting Metal Nanowires within Discrete Self-Assembled Peptide Nanotubes. Science. 2003;300 (5619):625627. doi: $10.1126 /$ science. 1082387

6. Shaon Kumar Das. Scope and Relevance of using Pesticide Mixtures in Crop Protection: A Critical Review. International Journal of Environmental Science and Toxicology.2014;2(5):119-123.

7. Shaon Kumar Das, Irani Mukherjee, Aman Kumar. Effect of soil type and organic manure on adsorption-desorption of flubendiamide. Environmental monitoring and assessment. 2015;187(7):403.

8. Pulickel M Ajayan. Nanotechnology: How does a nanofibre grow? Nature.2004;427:402-403. doi:10.1038/427402a

9. Shaon Kumar Das. Mode of action of pesticides and the novel trends-a critical review. International Research Journal of Agricultural Science and Soil Science. 2013;3(11):393-403.

10.Shaon Kumar Das. Recent development and future of Botanical pesticides in India. Popular Kheti. 2014;2(2):93-99.

11.Xia Y, Yang P, Sun Y, Wu Y, Mayers B, Gates B, et al. One dimensional nanostructures: synthesis, characterization and applications. Adv. Mater. 2003;15(5):353-389. doi: 10.1002/adma.200390087

12.Redl FX, Cho KS, Murray CB, Brien O. Three dimensional binary super lattices of magnetic nanocrystals and semiconductor quantum dots. Nature. 2003;423(6943):968-971. doi:10.1038/nature01702

13.Shaon Kumar Das, Irani Mukherjee. Effect of light and pH on persistence of flubendiamide. Bull Environ Contam Toxicol. 2011; 87(3): 292-296.

14.Mark A Poggi, Lawrence A Bottomley, Peter T Lillehei. Scanning probe microscopy. Anal. chem. 2002;74:2851-2862. doi: 10.1021/ac025695w

15.Kroto H W, Heath J R, O Brien S C, Curl R F, Smalley R E. This Week's Citation Classic $®$. Nature.1985;318:162-163.
16.T Jon Seiders, Kim K. Baldridge, Gunther H Grube, Jay S Siegel. Structure/Energy Correlation of Bowl Depth and Inversion Barrier in Corannulene Derivatives: Combined Experimental and Quantum Mechanical Analysis. J. Am. Chem. Soc. 2001;123:517-525. doi:10.1021/ja0019981

17.Shaon Kumar Das, Irani Mukherjee. Effect of moisture and organic manure on persistence of flubendiamide in soil. Bulletin of Environmental Contamination Toxicology. 2012;88(4):515-520.

18.Goverdhan Mehta and Gautam Panda. Quest for'Bucky-Balls' and'Bucky-Bowls': An Odyssey Through the Science of Organic Synthesis. Proc. Indian Natl. Sci. Acad.1998;A64:587-608.

19.U Deva Priyakumar, G Narahari Sastry. Heterobuckybowls: A Theoretical Study on the Structure, Bowl-to-Bowl Inversion Barrier, Bond Length Alternation, Structure-Inversion Barrier Relationship, Stability, and Synthetic Feasibility. J. Org. Chem. 2001;66(20):65236530.

20.Shaon Kumar Das, Irani Mukherjee, Aman Kumar. Effect of soil type and organic manure on adsorption-desorption of flubendiamide. Environmental Monitoring \& Assessment. 2015;187:403. doi:10.1007/s 10661-015-4623-2.

21.Shaon Kumar Das. Role of micronutrient in rice cultivation and management strategy in organic agriculture-A reappraisal. Agricultural Sciences. 2014;5:765-769.

22.Shaon Kumar Das. Recent developments in clean up techniques of pesticide residue analysis for toxicology study: a critical review. Universal Journal of Agricultural Research. 2014;2(6):198-202. doi: 10.13189/ujar.2014.020603

23.Wu M K, Windeler R S, Steiner C K R, Bors T, Friedlander S K. Controlled synthesis of nanosized particles by aerosol processes. Aerosol Sci. Technol. 1993;19(4):527-548. 